\title{
EVALUACIÓN AMBIENTAL ESTRATÉGICA APLICADA AL ABASTECIMIENTO DE ALIMENTOS Y SEGURIDAD ALIMENTARIA EN BOGOTÁ D.C.
}

\begin{abstract}
*Isabel Cristina Duarte Espinosa
Universidad Sergio Arboleda, Maestría en Gestión y Evaluación Ambiental, IDEASA- Medio Ambiente y

Sostenibilidad

https://orcid.org/0000-0002-3493-8377

icde27@gmail.com

**Carlos Alberto Echeverry Arciniegas
\end{abstract}

Universidad Sergio Arboleda, Maestría en Gestión y Evaluación Ambiental, IDEASA- Medio Ambiente y

Sostenibilidad

https://orcid.org/0000-0001-5774-0200

c_echeverry@hotmail.com

Para citar este artículo puede utilizar el siguiente formato:

Isabel Cristina Duarte Espinosa y Carlos Alberto Echeverry Arciniegas (2021): "Evaluación ambiental estratégica aplicada al abastecimiento de alimentos y seguridad alimentaria en Bogotá D.C.", Revista DELOS, Vol 14 № 38 (junio 2021). En línea:

https://www.eumed.net/es/revistas/delos/vol-14-no-38-junio-2021/abastecimiento-alimentos

\section{RESUMEN}

El Plan Maestro de Abastecimiento de Alimentos y Seguridad Alimentaria de Bogotá - PMASAB es el instrumento estructurante de primer nivel del Plan de Ordenamiento Territorial de Bogotá, que establece objetivos, políticas y estrategias de largo plazo para el abastecimiento y seguridad alimentaria de la ciudad. Debido a que la evaluación de mediano plazo realizada al PMASAB evidenció que su estrategia ambiental no fue evaluada ni implementada, sumado a que la Evaluación Ambiental Estratégica - EAE en Colombia se estancó en el 2010, este trabajo tiene por objetivo formular lineamientos y recomendaciones que permitan la incorporación de consideraciones ambientales en un próximo PMASAB. Lo anterior fue posible a partir de: 1) Aplicar la EAE Colombia del 2008 al PMASAB, 2) Analizar la visión de la EAE adoptadas por España y Chile para actualizar la EAE de Colombia, y 3) Analizar las posibles mejoras a nivel de Política, Plan y Programa en Bogotá, para la incorporación de consideraciones ambientales en el nuevo PMASAB. En consecuencia, la

\footnotetext{
*Microbióloga, Especialista en Diseño y Gestión Social de Tecnología de la Universidad de Los Andes. Especialista en Gerencia Ambiental y Gestión del Riesgo de Desastres y Magíster en Gestión y Evaluación Ambiental de la Universidad Sergio Arboleda.

**Tutor y Profesor Universitario. Ingeniero Civil, Especialista en Planeación Ambiental y Manejo Integral de los Recursos Naturales de la Universidad Militar Nueva Granada. Magíster en Ingeniería Civil de la Universidad de Los Andes. Asesor en asuntos ambientales de la Procuraduría General de la Nación en Colombia.
} 
propuesta aquí desarrollada se fundamentó en la aplicación del enfoque de pensamiento sistémico y la metodología de marco lógico. Como resultados, se evidenció que la aplicación de la EAE requiere fundamentalmente ajustarse al proceso de planeación propio a nivel país y a nivel ciudad. Así mismo, se observó que la visión de sostenibilidad a nivel país y de ciudad es amplia, poco clara, carente de lineamientos de política que puedan materializarse en apuestas estratégicas para el diseño de programas y proyectos que transformen el territorio.

PALABRAS CLAVE: Evaluación ambiental estratégica, seguridad alimentaria, pensamiento sistémico

\section{STRATEGIC ENVIRONMENTAL ASSESSMENT APPLIED TO FOOD SUPPLY AND FOOD SAFETY IN BOGOTÁ D.C.}

\section{ABSTRACT}

The Master Plan for Food Supply and Food Safety of Bogotá - PMASAB by its initials in Spanish, is the first level structuring instrument of the Bogota Land Management Plan, which establishes longterm objectives, policies and strategies for supply and safety food of the city. Due to the fact that the mid-term evaluation carried out to the PMASAB showed that its environmental strategy was not evaluated or implemented, added to the fact that the Strategic Environmental Evaluation - SEA in Colombia stalled in 2010, this work aims to formulate guidelines and recommendations that allow the incorporation of environmental considerations in an upcoming PMASAB. The above was possible from: 1) Applying the 2008 SEA Colombia to the PMASAB, 2) Analyzing the vision of the SEA adopted by Spain and Chile to update the SEA of Colombia, and 3) Analyzing the possible improvements at the Policy level, Plan and Program in Bogotá, for the incorporation of environmental considerations in the new PMASAB. Consequently, the proposal developed here was based on the application of the systems thinking approach and the logical framework methodology. As results, it was evidenced that the application of the SEA fundamentally requires adjusting to the planning process itself at the country level and at the city level. Likewise, it was observed that the vision of sustainability at the country and city level is broad, unclear, lacking policy guidelines that can materialize in strategic bets for the design of programs and projects that transform the territory.

KEY WORDS: Strategic environmental assessment, food security, systems thinking.

\section{INTRODUCCIÓN}

El Plan Maestro de Abastecimiento de Alimentos y Seguridad Alimentaria de BogotáPMASAB, adoptado a través del Decreto Distrital (DD 315/2006), es el instrumento estructurante de primer nivel del Plan de Ordenamiento Territorial - POT de Bogotá (DD 190/2004), que establece 
objetivos, políticas y estrategias de largo plazo en materia de abastecimiento y seguridad alimentaria del Distrito Capital (SDP, 2019). Así mismo, el PMASAB, plantea como estrategias ambientales su articulación con las estrategias del Plan Distrital de Gestión Ambiental - PGA (DD 061/2003 y DD 456/2008), en los componentes de investigación, educación, participación, fortalecimiento, coordinación, control, vigilancia y ubicación de la infraestructura. Con fundamento en la evaluación al PMASAB efectuada por (ICTA-UNAL, 2015), puede afirmarse que la estrategia ambiental no presenta evidencia de haber sido analizada o evaluada, y no guarda concordancia con el Documento Técnico de Soporte del PMASAB. Sumado a lo anterior, (ICTA-UNAL, 2015) identificó deficientes decisiones estratégicas ambientales, baja coherencia en el encadenamiento de política, plan y programa - PPP, en materia de seguridad alimentaria, lo cual conlleva a una baja ejecución del PMASAB desde las metas de corto plazo y una baja sostenibilidad de este.

Cabe mencionar que, para la formulación del PMASAB, a nivel nacional se estaban desarrollando los primeros ejercicios de Evaluación Ambiental Estratégica - EAE (MAVDT, 2008), como avance metodológico para incorporar consideraciones ambientales en las políticas públicas y planes más relevantes del país. Sin embargo, este proceso se estancó con las últimas EAE desarrolladas en el 2010, posterior a la publicación de la "Guía Práctica para Formular Evaluaciones Ambientales Estratégicas en Colombia" desarrollada en el 2008 para el Ministerio de Ambiente, Vivienda y Desarrollo Territorial de Colombia-MAVDT'.

En concordancia, (Gómez, 2011) indica que el sector público colombiano se caracteriza por la proliferación de políticas públicas que formulan y ejecutan una variedad de organismos sin suficiente coordinación, sumado a los vacíos normativos y la ausencia de estandarización sistemática entre áreas, sectores e instrumentos de planificación. A su vez, el autor indica que es habitual no encontrar diferencias entre PPPs, siendo esto evidente en el PMASAB, puesto que como Plan Maestro e instrumento del POT de Bogotá, aborda temáticas propias de las políticas públicas (ICTAUNAL, 2015). Así mismo, (Gómez, 2011) concluye que las políticas públicas en Colombia se ven afectadas en su eficacia y eficiencia haciendo de la planeación un campo diverso y confuso que condiciona los procedimientos y metodologías que se puedan desarrollar en el marco de la EAE.

En la actualidad, el Distrito Capital cuenta con la reciente "Política Pública de Seguridad Alimentaria y Nutricional para Bogotá: Construyendo Ciudadanía Alimentaria 2019-2031", CONPES D.C. 09/2019. Así mismo, la Alcaldía Mayor de Bogotá se encuentra en proceso de revisión del POT de Bogotá. Esta revisión se desarrolla involucrando lo propuesto por el actual Plan Distrital de Desarrollo - PDD "Nuevo contrato social y ambiental para el Siglo XXI 2020-2024" (Acuerdo Distrital 761/2020). Al cierre de este trabajo, el PMASAB aún se encuentra vigente y en espera de los resultados del proceso de adopción del nuevo POT de Bogotá (CONPES D.C. SAN, 2019), que de origen a un nuevo PMASAB. Del mismo modo, la Alcaldía ya cuenta con (SDDE y FAO, 2018) y con

\footnotetext{
${ }^{\dagger}$ Actualmente Ministerio de Ambiente y Desarrollo Sostenible-MADS
} 
(SDDE y Región Central, 2020), los cuales constituyen parte de los estudios de soporte para este nuevo PMASAB.

Dada la problemática identificada anteriormente, el presente trabajo tiene por objetivo formular lineamientos y recomendaciones que permitan la incorporación de consideraciones ambientales en un próximo PMASAB. Lo anterior, a partir de: 1) Aplicar la EAE Colombia del 2008 al PMASAB, 2) Analizar la visión de la EAE adoptadas por España y Chile para actualizar la EAE de Colombia, y 3) Analizar las posibles mejoras a nivel de PPP en Bogotá, para la incorporación de consideraciones ambientales en el nuevo PMASAB.

\section{METODOLOGÍA}

El presente estudio se fundamenta en un ejercicio de investigación aplicada, a un nivel de profundidad descriptivo y explicativo, de naturaleza cualitativa, fundamentado en la Metodología de Marco Lógico - MML y el uso de herramientas como la codificación abierta proveniente del diseño emergente, el análisis de actores y análisis de redes. En consecuencia, el alcance de los análisis en el presente trabajo se delimita a partir de: Los requerimientos planteados en cada una de las fases de los procesos de EAE (según Colombia, Chile y España), el proceso de planeación característico de Colombia y Bogotá D.C, según la normatividad que les es propia y las respuestas de carácter oficial emitidas por las entidades públicas competentes. En consecuencia, para el desarrollo del primer objetivo, el cual pretende aplicar la EAE planteada en (MAVDT, 2008) al PMASAB, la cual se describe en la Tabla 1. Esta metodología se basa en la revisión de documentos oficiales y la normativa adoptada, el diligenciamiento de plantillas y construcción de modelos propuestos a partir de la revisión documental oficial, para su posterior análisis. 
Tabla 1

Metodología Objetivo 1 según Guía EAE Colombia (MAVDT,2008)

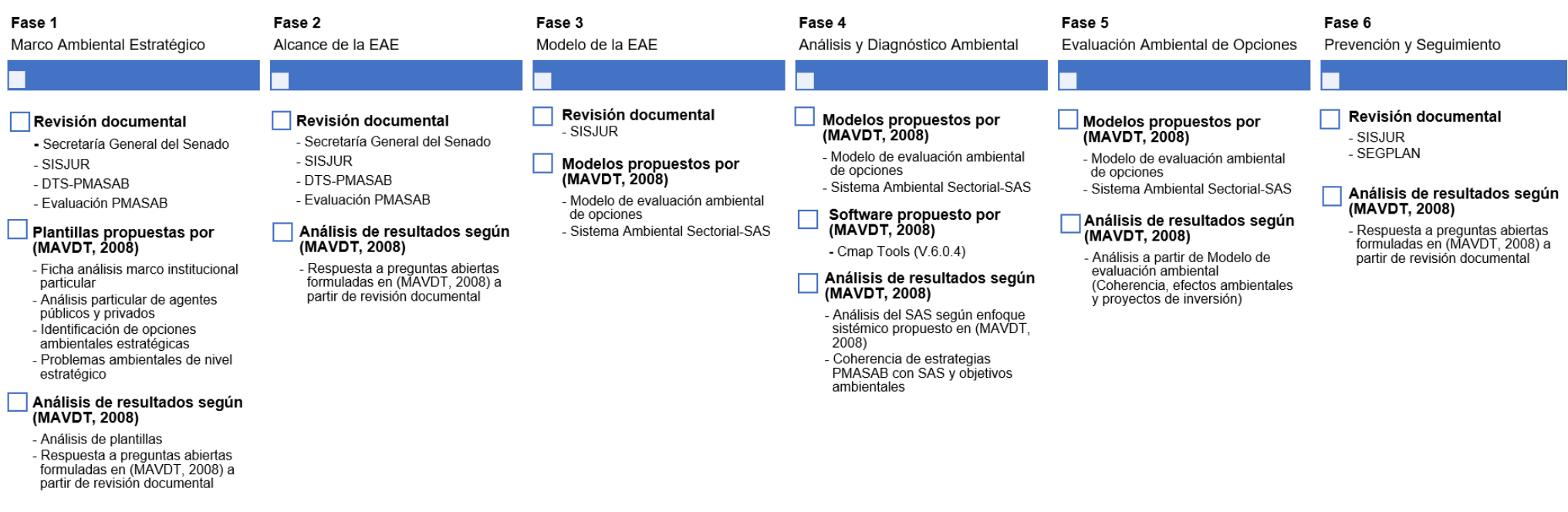

Fuente: Elaboración propia 
Para el desarrollo del segundo objetivo, el cual pretende analizar la visión de la EAE adoptada en España y Chile, se utilizó la metodología descrita en la Tabla 2, la cual involucra la revisión de bases de datos para la construcción del marco conceptual de la EAE, con el cual se desarrolla la codificación cualitativa abierta a los procedimientos de EAE de Colombia, España y Chile. Lo anterior, con el propósito de construir mapas mentales, para su análisis y posterior construcción de la propuesta de EAE actualizada para Colombia, ajustada al proceso de planeación del país.

\section{Tabla 2}

Metodología objetivo 2

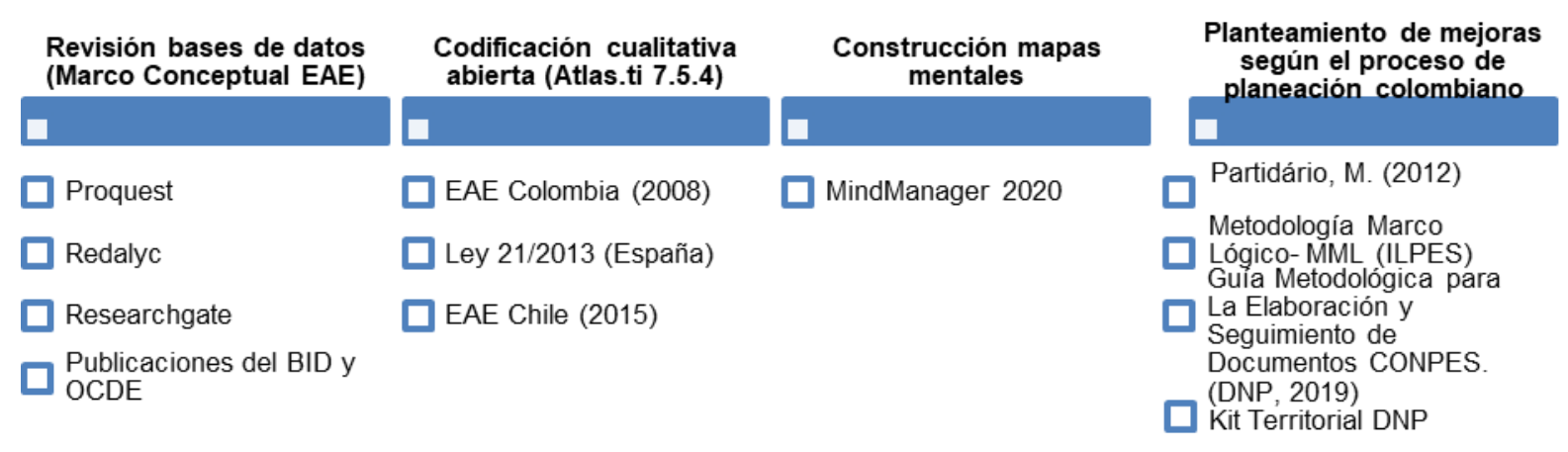

Fuente: Elaboración propia

Para el desarrollo del tercer objetivo, el cual pretende analizar las posibles mejoras a nivel de PPP, para la incorporación de consideraciones ambientales con respecto al abastecimiento de alimentos y la seguridad alimentaria en Bogotá, se utilizó la metodología descrita en la Tabla 3. Lo cual incluye la revisión documental del marco conceptual actual sobre abastecimiento y seguridad alimentaria, que involucre el marco conceptual adoptado a nivel país y nivel ciudad. Lo anterior, con el propósito de construir el diagnóstico a través del análisis de redes que permita proponer una $E A E$ ajustada a la construcción de un nuevo PMASAB, teniendo en cuenta las características del proceso de planeación de la ciudad.

\section{Tabla 3}

Metodología objetivo 3

$\begin{gathered}\text { Revisión documental (Marco } \\ \text { Conceptual Abastecimiento y } \\ \text { Seguridad Alimentaria) }\end{gathered}$
$\square$ FAO
$\square$ CGIAR
$\square$ Secretaría General del
Senado
$\square$ Documentos CONPES
• Seguridad alimentaria
•Cambio climático
• ODS

Revisión documental Alcaldía Mayor de Bogotá

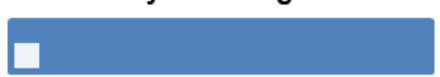

$$
\text { SISJUR }
$$

Actual proceso de revisión del POT Bogotá D.C

Políticas públicas distritales sectoriales vigentes

CONPES D.C

Diseño de cuestionario con

preguntas abiertas y

solicitud de respuesta oficial a la SDDE
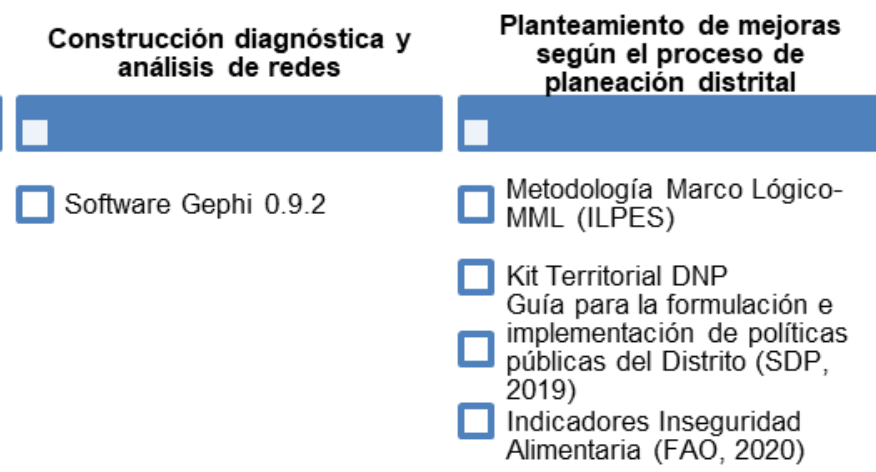
Fuente: Elaboración propia.

\section{RESULTADOS Y ANÁLISIS}

\section{Resultados y análisis de la aplicación de la EAE Colombia al PMASAB}

De la aplicación de la EAE Colombia propuesta en (MAVDT, 2008), se obtuvo el Sistema Ambiental Sectorial - SAS del PMASAB, compuesto por un modelo ambiental sectorial en conjunto con un mapa de dinámicas institucionales y de agentes. De su análisis se pudo evidenciar que:

Los mayores generadores de efectos ambientales en el SAS son: El Transporte de alimentos, la Central de Abastos de Bogotá - CORABASTOS, los hipermercados y supermercados, las plazas de mercado, la industria transformadora, junto con sus industrias complementarias y el consumidor final.

- Las principales problemáticas ambientales identificadas: Deficiente logística del transporte de alimentos, inadecuadas instalaciones, prácticas de manejo y almacenamiento de alimentos.

Ineficiente articulación de políticas ambientales tendientes al cumplimiento de la normatividad ambiental por parte de las plazas de mercado, concentración de la comercialización de alimentos en CORABASTOS, bajo desarrollo en materia de conservación y mejoramiento paisajístico de la ciudad.

Las estrategias operativas del PMASAB con mayor contribución a la estructura del SAS son: Las dirigidas a la conectividad del sistema alimentario, el acceso a la información en condiciones de equidad para el desarrollo de sistemas tecnológicos, la cultura de los operadores para apropiar prácticas modernas y la aplicación de nuevas tecnologías.

Se evidencia la necesidad de que el PMASAB se articule con otros instrumentos de su misma categoría, como es el Plan Maestro de Residuos Sólidos, el Plan Maestro de Movilidad.

Existe deficiente articulación de políticas ambientales tendientes a promover el cumplimiento de la normatividad y las estrategias de manejo ambiental, nutricional, de higiene y salubridad en las plazas de mercado distritales.

Aunque el PMASAB articuló sus estrategias ambientales con las estrategias del PGA 2001-2009, (ICTA-UNAL, 2015) evidenció las fallas de dicho PGA y la deficiente articulación PMASAB - PGA.

Así mismo, como resultado de la aplicación de la EAE propuesta por (MAVDT, 2008), se pudo establecer que el PMASAB no cuenta con recursos presupuestales para su ejecución, dado que se ejecuta a través de programas y proyectos de los PDDs. Esta dependencia afecta la planeación operativa del PMASAB, dando lugar a un bajo cumplimiento en sus metas. De igual forma, la incoherencia y vacío normativo respecto al ordenamiento territorial y uso del suelo se evidencian en la práctica, a través de la coexistencia de usos incompatibles del suelo, sumado a la desarticulación del PMASAB con el POT de Bogotá, respecto a la integración con la Estructura Ecológica Principal - 
EEP de la ciudad. Aunado a lo anterior, el PMASAB no cuenta con instrumentos de planeamiento para el área rural y no contempla la figura de Unidad de Planeación Rural planteada por el POT de Bogotá.

De igual forma, se identificó que el mecanismo normativo de seguimiento a los planes maestros del Distrito Capital, es un instrumento ineficiente, sin retroalimentación alguna, el cual exige evaluación de impactos ambientales, desconociendo que el PMASAB no contó con un análisis ambiental y que los proyectos asociados a este tampoco cuentan con evaluaciones de este tipo. Así mismo, CORABASTOS y el mercado mayorista no fueron tenidos en cuenta en el sistema de abastecimiento planteado por el PMASAB. Sumado a lo anterior, la adopción del PMASAB con antelación a la Política Distrital de Seguridad Alimentaria y Nutricional 2007-2015- PDSAN, generó una baja participación institucional del Consejo Directivo del PMASAB. Todo lo anterior, ocasionó que los agentes más afectados por el PMASAB fueran las organizaciones campesinas y las gobernaciones de los departamentos de Boyacá, Cundinamarca, Meta y Tolima, que conforman junto con Bogotá D.C la Región Central de Colombia. Lo anterior, dado que organizaciones campesinas y gobernaciones requieren mayor atención porque no son tenidas en cuenta en la ejecución del PMASAB, pero si pueden obstruir el desarrollo de este (ICTA-UNAL, 2015).

\section{Resultados y análisis de la visión de la EAE adoptadas por España y Chile para la incorporación de consideraciones ambientales en el contexto colombiano}

A partir de los mapas mentales construidos, se evidenció una mayor correspondencia entre la EAE simplificada de España con el proceso de EAE colombiano planteado por (MAVDT, 2008). Del mismo modo, fue posible establecer que la EAE en España cuenta con un reducido número de momentos de participación oficiales en comparación con Chile y Colombia. De igual forma, se encuentra fuertemente relacionado con la evaluación de impactos ambientales. En contraste, la EAE de Chile, plantea la construcción de un diagnóstico ambiental más robusto que el planteado por España y Colombia, al igual que desarrolla una etapa continua que evalúa el cumplimiento de los objetivos de la EAE en la ejecución de los PPP, a partir de la definición de Factores Críticos de Decisión-FCD. Lo anterior, evidencia la fuerte relación de la EAE de Chile con el modelo centrado de decisión indicado por (Partidário, 2012).

En contraposición a la EAE de España y Chile, la EAE de Colombia evalúa alternativas a nivel de objetivos, estrategias y acciones operativas, haciendo el procedimiento mucho más dispendioso y disperso, con respecto al nivel estratégico el cual es el objeto de la evaluación de alternativas para España y Chile.

En consecuencia, se planteó la propuesta de EAE actualizada para Colombia, la cual cuenta con 7 fases y 3 momentos de participación en cuanto a estrategia de validación, como se describe en la Tabla 4. Allí mismo, se encuentran especificadas las actividades cuyos resultados son validados y 
complementados de forma participativa en los momentos de participación correspondientes, al igual que la relación entre las actividades del procedimiento.

\section{Tabla 4}

Estructura de la propuesta de EAE para Colombia

\begin{tabular}{|c|c|c|}
\hline Fases y Actividades EAE & Validación & $\begin{array}{l}\text { Relación Entre } \\
\text { Actividades }\end{array}$ \\
\hline \multicolumn{3}{|c|}{ Fase 1. Enfoque de la EAE } \\
\hline \multicolumn{3}{|l|}{ 1.1 Definir el problema que pretende solucionar la PPP } \\
\hline \multicolumn{3}{|l|}{ 1.2 Definir alcance y contenido de la PPP propuesta } \\
\hline \multicolumn{3}{|l|}{ 1.3 Describir el objeto de evaluación de la EAE } \\
\hline \multicolumn{3}{|l|}{ 1.4 Identificar objetivos estratégicos de la PPP } \\
\hline 1.5 Definir objetivo (s) de la EAE & $\begin{array}{c}\text { 1er momento de participación } \\
\text { (Actividad 1.5) }\end{array}$ & \\
\hline \multicolumn{3}{|c|}{ Fase 2. Marco Ambiental Estratégico } \\
\hline 2.1 Establecer el marco institucional & & Insumo de 4.1 y de 5.3 \\
\hline 2.2 Establecer el marco de agentes & $\begin{array}{l}\text { 2do momento de participación } \\
\text { (Actividad 4.4) }\end{array}$ & $\begin{array}{l}\text { Insumo de } 4.1,4.2 \text { y } \\
\text { de } 5.3\end{array}$ \\
\hline $\begin{array}{l}\text { 2.3 Describir el proceso de decisión y sincronización entre este y la } \\
\text { EAE }\end{array}$ & & Insumo de 5.3 \\
\hline 2.4 Definir el marco del problema ambiental & $\begin{array}{l}\text { 2do momento de participación } \\
\text { (Actividad 4.4) }\end{array}$ & Insumo de 4.1 y de 5.3 \\
\hline 2.5 Identificación de objetivos ambientales & & Insumo de 4.1 y de 5.3 \\
\hline \multicolumn{3}{|l|}{ 2.6 Necesidades de información } \\
\hline \multicolumn{3}{|c|}{ Fase 3. Modelo de la EAE } \\
\hline \multicolumn{3}{|l|}{$\begin{array}{l}\text { 3.1 Identificar metodologías y herramientas a usar en cada una de } \\
\text { las actividades que hacen parte de las fases de elaboración de la } \\
\text { EAE }\end{array}$} \\
\hline \multicolumn{3}{|l|}{ 3.2 Definir herramientas de evaluación y análisis } \\
\hline \multicolumn{3}{|c|}{ Fase 4. Análisis y Diagnóstico Ambiental } \\
\hline 4.1 Recopilación de insumos para la construcción de la red & & $\begin{array}{l}\text { A partir de 2.1, 2.2, } 2.4 \\
\text { y } 2.5\end{array}$ \\
\hline 4.2 Establecimiento de relaciones en la red & $\begin{array}{l}\text { 2do momento de participación } \\
\text { (Actividad 4.4) }\end{array}$ & $\begin{array}{l}\text { Se construye a partir } \\
\text { de } 2.2\end{array}$ \\
\hline \multicolumn{3}{|l|}{ 4.3 Construcción de la red (SAS) } \\
\hline 4.4 Validación de la primera revisión de la red & & \begin{tabular}{|l|} 
A partir de $4.1,4.2 y$ \\
4.3 Insumo de 4.5 \\
\end{tabular} \\
\hline \multicolumn{3}{|l|}{ 4.5 Construcción de una nueva versión de red (SAS mejorado) } \\
\hline \multicolumn{3}{|c|}{ Fase 5. Evaluación Ambiental de Opciones } \\
\hline \multicolumn{3}{|l|}{$\begin{array}{l}5.1 \text { Evaluar que los objetivos del PPP permitan la incorporación de } \\
\text { problemas ambientales }\end{array}$} \\
\hline \multicolumn{3}{|l|}{$\begin{array}{l}5.2 \text { Revisar coherencia y articulación de los objetivos del PPP con } \\
\text { las estrategias del PPP }\end{array}$} \\
\hline 5.3 Análisis de estrategias de la PPP & & \begin{tabular}{|l|} 
Se realiza a partir de \\
$2.1,2.2,2.3,2.4$ y 2.5 \\
\end{tabular} \\
\hline $\begin{array}{l}5.4 \text { Complementar estrategias del PPP para que incorporen } \\
\text { aspectos ambientales }\end{array}$ & $\begin{array}{l}\text { 3er momento de participación } \\
\text { (Actividad 7.1) }\end{array}$ & \\
\hline \multicolumn{3}{|l|}{$\begin{array}{l}5.5 \text { Analizar la planeación operativa con los objetivos y estrategias } \\
\text { ajustadas ambientalmente del PPP }\end{array}$} \\
\hline $\begin{array}{l}5.6 \text { Plantear alternativas operativas ambientales para las acciones } \\
\text { operativas que permitan el involucramiento de problemáticas } \\
\text { ambientales }\end{array}$ & $\begin{array}{l}\text { 3er momento de participación } \\
\text { (Actividad 7.1) }\end{array}$ & \\
\hline \multicolumn{3}{|l|}{$\begin{array}{l}5.7 \text { Analizar las alternativas operativas ambientales a partir de } \\
\text { normas, agentes, aspectos financieros, económicos y sociales }\end{array}$} \\
\hline \multicolumn{3}{|c|}{ Fase 6. Prevención y Seguimiento } \\
\hline $\begin{array}{l}\text { 6.1 Construcción del marco seguimiento enfocado en instrumentos } \\
\text { de gestión ambiental del sector de aplicación de la PPP }\end{array}$ & & \\
\hline
\end{tabular}




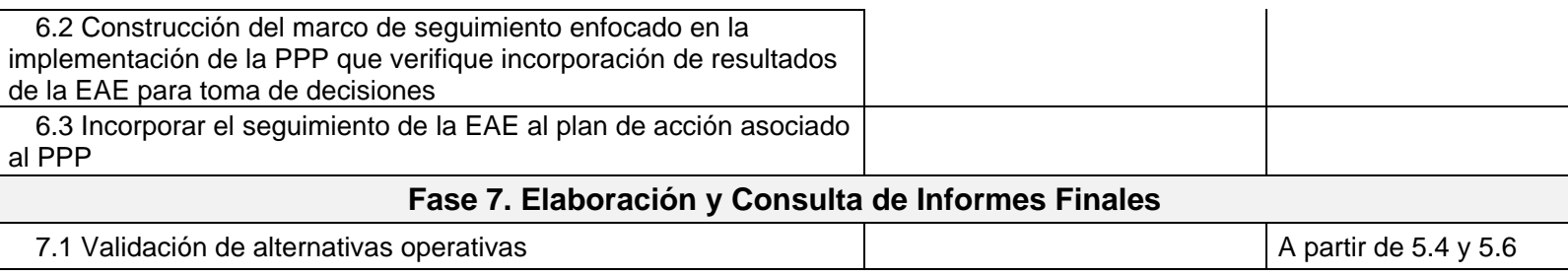

Nota. PPP - Política/plan/programa. SAS - Sistema Ambiental Sectorial. Fuente: Elaboración propia

Dentro de las mejoras propuestas para la EAE Colombia se destacan:

- La incorporación de una fase inicial que permita el análisis del instrumento de decisión y el establecimiento del alcance de la EAE.

La redefinición del marco ambiental estratégico, que involucre aspectos naturales, económicos, sociales, culturales, patrimonio natural y cultural, descripción de problemas y preocupaciones ambientales y descripción de conflictos socioambientales según lo planteado por (España, 2013) y (MMA, 2015a). Así mismo, se incorporan como marco de los objetivos ambientales, los lineamientos de política pública en el CONPES 3700/2011 sobre Cambio Climático y el CONPES 3918/2018 sobre Objetivos de Desarrollo Sostenible - ODS como visión del proceso de EAE.

- $\quad$ En la fase 3 las herramientas y las metodologías seleccionadas para llevar a cabo la EAE deben enmarcarse en el enfoque sistémico de sostenibilidad según (Gallopín, 2003).

Respecto a la Fase 4 sobre análisis y diagnóstico de la red, se propone la elaboración del SAS por medio del software Gephi debido a que el programa permite construir la red y cuenta con herramientas para el análisis de redes.

En la Fase 5 sobre evaluación ambiental de opciones, se plantea evaluar la articulación de objetivos-estrategias-acciones operativas y se proponen definir alternativas operativas ambientales para las acciones operativas, con miras a identificar y evaluar parcialmente alternativas operativas ambientales que guíen la implementación de acciones a nivel de proyectos.

- $\quad$ Como propuesta para la Fase 6 de prevención y seguimiento, se instala e inicia la etapa continua que le permita a la EAE evaluar la incorporación de lineamientos y objetivos ambientales durante la implementación de la PPP. De igual forma se propone que, en los planes de acción de las PPP del nivel nacional, se incluya el seguimiento a la EAE.

\section{Resultados y análisis a posibles mejoras para la incorporación de consideraciones ambientales en el abastecimiento de alimentos y seguridad alimentaria de Bogotá D.C.}

Al analizar la actual política de seguridad alimentaria distrital (CONPES D.C 09/2019), a la luz del marco conceptual sobre sistemas alimentarios sostenibles desarrollado por (CGIAR, 2020), se observó que dicho CONPES D.C se encuentra mayormente centrada en el consumo y débilmente en la preproducción, producción y cadena de abastecimiento

Así mismo, se construyó el SAS y a través de las herramientas de análisis de redes disponibles en Gephi, se obtuvo una red altamente fragmentada, dónde se presentan una alta 
modularidad que evidencia la forma superficial en que se abordan problemáticas persistentes de la seguridad alimentaria y el papel de las entidades distritales en la red (Figura 1). De estos análisis fue posible establecer que:

- $\quad$ EI SAS evidencia los roles estratégicos de la Empresa de Acueducto y Alcantarillado de Bogotá-EAAB y de CORABASTOS en la red. La EAAB aparece, debido a la relevancia en la relación acceso al agua potable y la seguridad alimentaria, mientras que el papel de CORABASTOS es preponderante debido a que concentra la entrada de alimento a la ciudad.

- Las instituciones gubernamentales del Distrito Capital se encuentran totalmente desligadas de la red debido al bajo nivel de articulación con los actores y las dinámicas de la red. Sin embargo, se estiman como los actores con mayores posibilidades de realizar conexiones.

- Las problemáticas identificadas en el SAS que deben priorizarse en las intervenciones son: La deficiencia en acceso a servicios básicos, el escaso porcentaje de prácticas de autoconsumo, el alto precio de los alimentos, los temas relacionados con la ruralidad de Bogotá y la reducción de la inseguridad alimentaria. Esta última, evidencia el bajo compromiso de las intervenciones planteadas para lograr un impacto en la reducción de la inseguridad alimentaria. De igual forma, en materia ambiental, se destacan como prioritarios el inadecuado manejo de residuos y los altos porcentajes de desechos posconsumo.

- Se identificaron como temas de integración en la red: El acceso al servicio de acueducto, las estrategias centradas en la reducción de los indicadores de inseguridad alimentaria y lo relacionado con la ruralidad bogotana.

El análisis de red indica que se equipara el papel de la ruralidad de Bogotá con el de la Región Central, lo cual niega la importancia del ecosistema de páramo que reside en el Distrito Capital. 


\section{Figura 1}

Espacialidad de la red
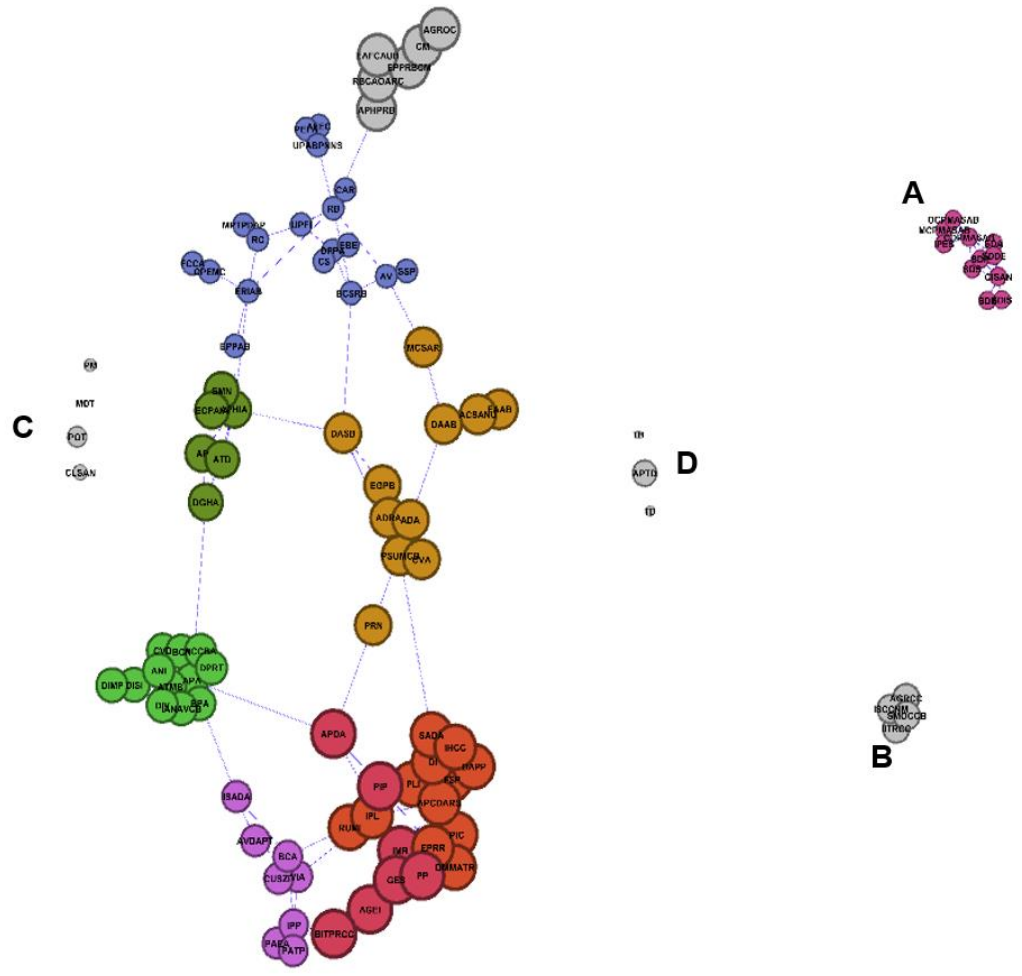

Nota. A - Modulo conformado por entidades distritales, B - Modulo problemáticas de actividades ganaderas, C - Modulo sobre Plazas de Mercado, POT vigente, y modelo de distribución tradicional, D - Modulo de temáticas relacionadas con tiendas de barrio. Fuente: Elaboración propia.

En consecuencia, a partir del análisis de redes aquí desarrollado, se pudo evidenciar las discrepancias entre los marcos conceptuales abordados en los diferentes estudios contratados como soporte técnico del nuevo PMASAB y el CONPES D.C 09/2019. Así mismo, se identificaron problemáticas desarrolladas en el diagnóstico, que no cuentan con una intervención en la estructura programática propuesta por los estudios soportes del nuevo PMASAB, al igual que se identificaron temáticas que cuentan con apuestas programáticas, pero nunca fueron descritas en el diagnóstico.

Tal es el caso del aumento en el precio de los alimentos debido a las ineficiencias del sistema de abastecimiento que afecta la canasta básica familiar, la cual no es analizada. Así mismo, el diagnóstico del CONPES D.C 09/2019, involucra el sistema de abastecimiento de alimentos y el agua, pero esta última no es tenida en cuenta en los estudios soporte del nuevo PMASAB. De igual 
forma, los estudios soporte identifican la necesidad del mejoramiento del mercado mayorista pero no cuenta con una apuesta estratégica para esto. El nuevo PMASAB se centra en las actividades logísticas, pero no cuenta con información sobre los efectos ambientales generados por estas, que sustenten la propuesta programática y la posterior toma de decisiones. Del mismo modo, se evidenció dentro de la propuesta programática del nuevo PMASAB, un programa sobre diversificación productiva, recuperación de productos originarios y de saberes tradicionales, al igual que la articulación con la Región Central respecto a la gestión de riesgos de desastres de origen hidroclimático. Lo anterior, evidencia la necesidad de contextualizar en el diagnóstico temas de agrobiodiversidad según lo planteado por (Bioversity International, 2017) y le inseguridad alimentaria desde el enfoque de gestión del riesgo de desastres según (PESA, 2011).

También, se hace énfasis en la necesidad de plantear estrategias efectivas en la estructura programática, que desincentiven el cultivo de papa y la actividad ganadera que se desarrolla en el páramo de la ruralidad de Bogotá, actividades que tienen alto impacto en estos ecosistemas. De igual forma, se requieren estrategias que le den cumplimiento a lo adoptado normativamente respecto a la agricultura urbana y periurbana para el Distrito Capital, que involucra investigación, transferencia de conocimiento y comercialización

En efecto, el análisis de redes realizado en este trabajo, si bien es utilizado a nivel de análisis y diagnóstico del problema, puede revisarse desde la perspectiva de los sistemas socio-ecológicosinfraestructurales. Dicho enfoque implica la integración de las relaciones socio ecológicas con la infraestructura, los actores y las instituciones (Armendáriz et al., 2016). Al revisar el modelo marco de los sistemas alimentarios urbanos planteado en (Armendáriz et al., 2016), es posible señalar que el SAS aquí obtenido carece de aspectos relacionados con: Tecnologías disponibles, el empleo relacionado con el crecimiento económico, la huella ecológica de las actividades del sistema, la capacidad de carga del territorio, la presión de las actividades del sistema sobre el espacio urbano, entre otros.

En consecuencia, se construyó la propuesta de EAE ajustada al nuevo PMASAB, a partir del diagnóstico y los aspectos relacionados con la planeación propia del Distrito Capital y su ciclo de políticas públicas. Esta propuesta está dirigida a que el PMASAB busque la construcción de un Sistema Alimentario Sostenible. Por lo cual, a nivel procedimental, se mantiene la estructura propuesta para la EAE Colombia, planteada en la Tabla 4. Entre los ajustes realizados puntualmente para la EAE del nuevo PMASAB se encuentran:

Se propone el análisis de problemáticas y preocupaciones ambientales del informe GEO (MMA, 2015b), con respecto a políticas públicas del Distrito Capital vigentes y la disponibilidad de información espacial, relacionadas con abastecimiento y seguridad alimentaria (Tabla 4, Actividad 2.5). 
- Se hace especial énfasis en las necesidades de construir información espacial respecto a la inseguridad alimentaria en el Distrito Capital.

- $\quad$ Para la Fase 5 de la EAE, se propone ahondar en la articulación con el PGA 20082038, el CONPES D.C. 09/2019, la visión de sostenibilidad y al menos los ODS 1, 2 y 13, como indica (FAO, 2019).

Para la Fase 6, se plantea la articulación con otros instrumentos de gestión ambiental distrital como es el Plan de Acción Cuatrienal Ambiental-PACA ligado a los PDDs.

Se plantea la creación de planes de acción para los planes maestros y la incorporación del seguimiento de la EAE a este instrumento.

Así mismo, debido a que el PMASAB se ejecuta a través de PDDs, se realiza una propuesta de articulación entre políticas públicas vigentes del Distrito Capital, nuevo PMASAB y PDDs, como se indica en la Figura 2.

\section{Figura 2}

Marco conceptual de la articulación del nuevo PMASAB con políticas públicas, nuevo POT de Bogotá y PDDs

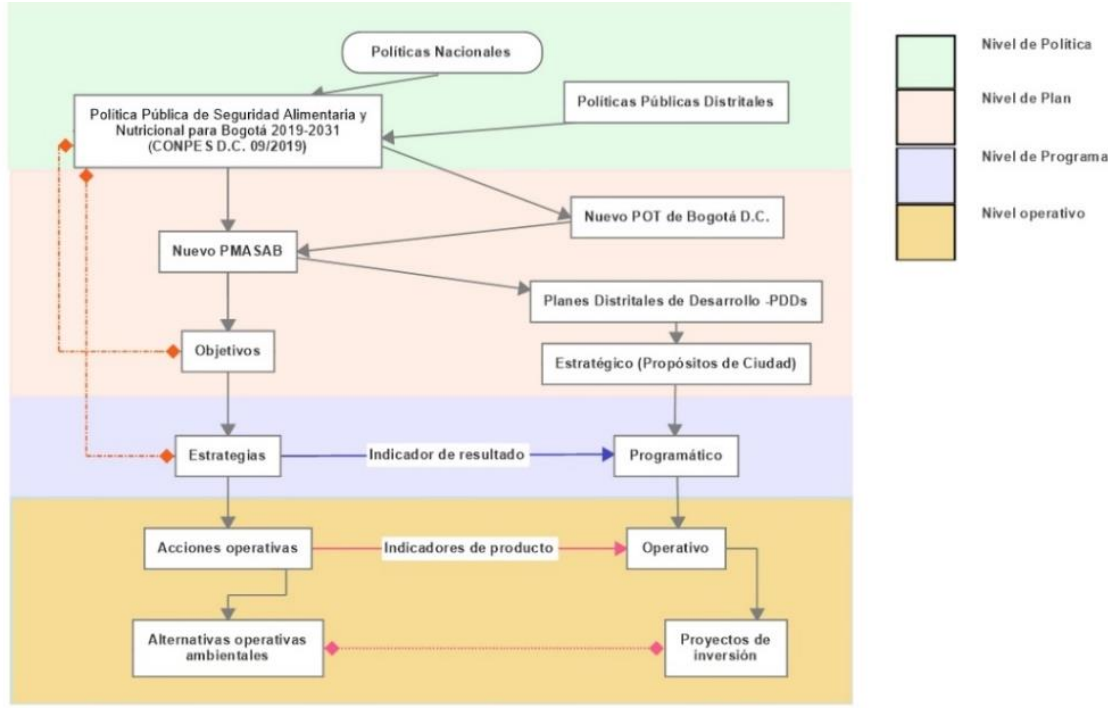

Fuente: Elaboración propia.

De esta forma, se plantea una articulación a nivel de objetivo y estrategias entre nuevo PMASAB y CONPES D.C. 09/2019 y una articulación a nivel de estrategias del nuevo PMASAB con programas del PDD a través de indicadores de resultado. Por último, la articulación entre alternativas operativas ambientales del nuevo PMASAB con proyectos de inversión del PDD, se da a través de indicadores de producto, en concordancia con la MML descrita en (ILPES, 2005). 


\section{CONCLUSIONES}

A través de la aplicación de la EAE Colombia al caso particular del PMASAB fue posible evidenciar que instrumentos como este no cuentan con recursos presupuestales propios, por lo cual se ejecuta a través de programas y proyectos de los PDDs. Esta dependencia afecta la planeación operativa del PMASAB, dando lugar a un bajo cumplimiento en sus metas. Así mismo, se evidencia incoherencia y vacío normativo respecto al ordenamiento territorial y uso del suelo, en la coexistencia de usos incompatibles del suelo generada por equipamientos de abastecimiento de alimentos y la desarticulación entre el PMASAB y el POT de Bogotá. respecto a la integración con la EEP de la ciudad. A su vez, existe deficiente articulación de políticas ambientales tendientes a promover el cumplimiento de la normatividad y las estrategias de manejo ambiental, nutricional, de higiene y salubridad en las plazas de mercado distritales. También, se observa un deficiente sistema de seguimiento y evaluación de la ejecución del PMASAB debido a la ineficiencia del instrumento dispuesto para esto.

Como conclusiones sobre la mejora de la EAE Colombia a partir de la EAE de España y Chile, se destaca que el cambio climático es tema obligatorio para España, mientras que la sostenibilidad es la visión del proceso de EAE en Chile. Dado lo anterior, este trabajo propone como marco de los objetivos ambientales lo planteado por el CONPES 3700/2011 sobre Cambio Climático y el CONPES 3918/2018 sobre ODS.

Así mismo, se estableció que la EAE en Chile, se encuentra ajustada fuertemente a los lineamientos del enfoque centrado en la decisión, del cual se destaca la inclusión de la etapa continua, la participación y la inclusión de FCD. Estos, surgen de la planeación por escenarios, que involucra un análisis prospectivo y de tendencias que no coincide con el proceso de planeación colombiano.

En consecuencia, la propuesta para la EAE Colombia, contempla el ajuste del proceso de EAE propuesto en (MAVDT, 2008), a las características normativas y al proceso de planeación del país, lo cual incluye la aplicación de las herramientas ofrecidas por la MML que involucran la identificación de actores y la identificación del problema a resolver. Por lo tanto, la propuesta metodológica de EAE para Colombia, está compuesta por 7 fases y 3 momentos de participación para validar objetivos de la EAE, validar y robustecer la construcción del SAS y validar estrategias y alternativas operativas ambientales. Adicionalmente, incorpora una fase de prevención y seguimiento que se mantiene durante la implementación de la PPP.

Con respecto a la incorporación de consideraciones ambientales en el nuevo PMASAB, se mantuvo la propuesta desarrollada de EAE para Colombia, ajustada al proceso de planeación del Distrito Capital, su ciclo de políticas públicas y a las características propias de la seguridad alimentaria de la ciudad. Lo anterior fue posible a partir del análisis de redes aquí desarrollado, con lo cual se pueden evidenciar las discrepancias entre los marcos conceptuales abordados en los 
diferentes estudios contratados como soporte técnico del nuevo PMASAB y el CONPES D.C 09/2019. Del mismo modo, se pudo evidenciar la baja coherencia entre el diagnóstico de las problemáticas y la propuesta de política pública para superar las mismas.

En efecto, es posible afirmar que el análisis de redes es una herramienta que puede contribuir al mejoramiento de la planeación y la toma de decisiones. Así mismo, la red es una herramienta útil para ser presentada a los actores y ser complementada por estos como estrategia de validación. De igual forma, la red permitiría evaluar la solución de política planteada por el CONPES D.C. 09/2019, con lo cual se podría determinar si las estrategias propuestas fortalecen la red o promueven su desarticulación. A partir de lo anterior, se obtendría una herramienta de consulta que puede ser actualizada y analizada no solo en el proceso de formulación, sino también en el proceso de toma de decisiones, durante la ejecución.

Respecto al objetivo planteado en este trabajo, se concluye que es posible incorporar consideraciones ambientales a instrumentos como el PMASAB, teniendo en cuenta como fundamental las características del proceso de planeación. De igual forma, es necesario indicar que el proceso de formulación de la PPP y el desarrollo de la EAE, deben diseñar un proceso de decisión en conjunto para el instrumento de decisión, según las particularidades del proceso de planeación. Así mismo, se destaca la necesidad de información espacial a escalas apropiadas, georreferenciada, que permita establecer la interacción de las actividades del sector con la EEP de la ciudad.

A su vez, se resalta la dificultad encontrada para implementar en Colombia el modelo centrado en la decisión estratégica. Esta dificultad se fundamenta en que dicho modelo plantea los FCD, los cuales surgen de la planeación por escenarios, mientras que, la planeación en Colombia se fundamenta en un único escenario futuro deseado. Lo anterior, entonces debe ser enfocado por la visión de sostenibilidad a nivel nacional y de ciudad, la cual aún es amplia, poco clara, carente de lineamientos de política que se materialicen en apuestas estratégicas a partir de las cuales se diseñen programas y proyectos que generen una transformación en el territorio. Por esta razón, cobra mayor relevancia la aplicación del enfoque de pensamiento sistémico.

Finalmente, como recomendaciones, se destaca la necesidad de realizar una mejora sustancial de la comprensión de las dinámicas de las problemáticas y sus relaciones causales. Así mismo, resulta imperativo revisar y estructurar el modelo sostenible de país y de ciudad, lo cual inicia con la comprensión del contexto de desarrollo, partiendo del análisis sistémico de la sostenibilidad como lo plantea (Gallopín, 2003).En cuanto al Distrito Capital, se recomienda trabajar en estrategias dirigidas a lograr que los actores se responsabilicen en el proceso de EAE, reconociendo que existe información generada y administrada por actores privados, asegurando una articulación regional eficiente y clara, la cual requiere la mejora de la capacidad institucional de las entidades distritales relacionadas. De igual forma, se debe trabajar en conocer las preocupaciones ambientales y conflictos socio ambientales relacionados con el sistema de abastecimiento de la ciudad. 
Como trabajos posteriores se plantea:

- $\quad$ El análisis sistémico de temáticas y sectores específicos a la luz de las orientaciones e implicaciones del cumplimiento de las orientaciones de política pública de los CONPES 3700/2011 y $3918 / 2018$.

- $\quad$ La recopilación de información espacial del Distrito Capital para la caracterización de la inseguridad alimentaria de la ciudad. Lo anterior involucra la inclusión de la agrobiodiversidad y de la inseguridad alimentaria como factor de vulnerabilidad.

- $\quad$ Partiendo de lo anterior, se plantea la identificación y la cuantificación, hasta donde sea posible, de todas las variables del sistema de abastecimiento de la ciudad, lo cual permitiría estructurar diferentes modelos de sostenibilidad.

- Se recomienda desarrollar el análisis de redes desde la perspectiva de los sistemas socio-ecológicos-infraestructurales de (Armendáriz et. al, 2016) que puede facilitar la articulación de la infraestructura planteada por el actual PDD para el sistema de abastecimiento alimentario.

\section{BIBLIOGRAFÍA}

Acuerdo Distrital 761 de 2020. (2020, 11 de junio). Concejo de Bogotá. Registro Distrital No. 6833. https://www.alcaldiabogota.gov.co/sisjur/normas/Norma1.jsp?i=93649

Armendáriz, V., Armenia, S., y Atzori, A. S. (2016). Systemic analysis of food supply and distribution systems in city-region systems-An examination of FAO's policy guidelines towards sustainable agri-food systems. Agriculture, 6(4). https://doi.org/10.3390/agriculture6040065

Bioversity International. (2017). To manage agrobiodiversity, we need to measure it: The three pillars of the Agrobiodiversity Index. https://www.bioversityinternational.org/ABD-Index/

CGIAR. (2020). Innovations for Sustainable Food Systems. Farming First. https://farmingfirst.org/foodsystems\#home

CONPES 3700 de 2011. (2011, 14 de julio). Estrategia Institucional para la Articulación de Políticas y Acciones en Materia de Cambio Climático en Colombia. https://colaboracion.dnp.gov.co/CDT/Conpes/Econ\%C3\%B3micos/3700.pdf

CONPES 3918 de 2018. (2018, 15 de marzo). Estrategia para La Implementación de Los Objetivos de Desarrollo Sostenible (ODS) en Colombia. https://colaboracion.dnp.gov.co/CDT/Conpes/Econ\%C3\%B3micos/3918.pdf

CONPES D.C 09 de 2019. (2019, 20 de diciembre). Política Pública de Seguridad Alimentaria y Nutricional para Bogotá: Construyendo Ciudadanía Alimentaria 2019-2031. http://sdp.gov.co/gestion-socioeconomica/conpes-dc/documentos-conpes-dc/conpes-dcaprobados

Decreto Distrital 061 de 2003. (2003, 13 de marzo). Alcaldía Mayor de Bogotá. Registro Distrital No.2832. https://www.alcaldiabogota.gov.co/sisjur/normas/Norma1.jsp?i=7196\&dt=S

Decreto Distrital 190 de 2004. (2004, 22 de septiembre). Alcaldía Mayor de Bogotá. Registro Distrital No. 3122. https://www.alcaldiabogota.gov.co/sisjur/normas/Norma1.jsp?i=13935\&dt=S

Decreto Distrital 315 de 2006. (2006, 18 de agosto). Alcaldía Mayor de Bogotá. Registro Distrital No. 
3596. https://www.alcaldiabogota.gov.co/sisjur/normas/Norma1.jsp?i=21063\&dt=S

Decreto Distrital 456 de 2008 (2008, 23 de diciembre). Alcaldía Mayor de Bogotá. Registro Distrital No.4124. https://www.alcaldiabogota.gov.co/sisjur/normas/Norma1.jsp?i=34284

Ley 21 de 2013. (2013, 9 de diciembre). Boletín Oficial Del Estado, 1-86. https://www.boe.es/buscar/pdf/2013/BOE-A-2013-12913-consolidado.pdf

FAO. (2019). Transformar los sistemas alimentarios para alcanzar los ODS. 2030/Alimentación, agricultura $y$ desarrollo rural en América Latina $y$ el Caribe (12). http://www.fao.org/publications/es

Gallopín, G. (2003). A systems approach to sustainability and sustainable. UN, ECLAC, Sustainable Development and Human Settlements Division. https://repositorio.cepal.org/handle/11362/5759

Gómez, J. (2011). La Evaluación Ambiental Estratégica (EAE) en Colombia: su adopción, criterios para la estructuración de su procedimiento administrativo y su aplicación a los planes de ordenamiento territorial. Revista Científica, (12). https://doi.org/10.14483/23448350.429

ICTA-UNAL. (2015). Evaluación al Plan Maestro de Abastecimiento y Seguridad Alimentaria de Bogotá. (223).

ILPES. (2005). Metodología general de identificación, preparación y evaluación de proyectos de inversión pública (Vol. 39). Santiago de Chile.

MAVDT. (2008). Guía de evaluación ambiental estratégica. https://www.minambiente.gov.co/images/AsuntosambientalesySectorialyUrbana/pdf/Evaluaci\%C 3\%B3n_Ambiental_Estrategica/Guia_de_evaluci\%C3\%B3n_ambiental_Estrat\%C3\%A9gica.pdf

MMA. (2015a). Guía de orientación para el uso de la evaluación ambiental estratégica en Chile. 75. https://mma.gob.cl/wp-content/uploads/2015/12/Guia-de-orientacion-para-la-eae-en-Chile.pdf

MMA. (2015b). Guía de orientación para incorporar la dimensión ambiental en procesos de ordenamiento territorial sustentable. http://biblioteca.digital.gob.cl/handle/123456789/1412

Partidário, M. D. R. (2012). Strategic Environmental Assessment Better Practice Guidemethodological guidance for strategic thinking in SEA. https://www.mspplatform.eu/practices/strategic-environmental-assessment-better-practice-guide-0

PESA. (2011). Seguridad alimentaria y nutricional -Conceptos Básicos. Proyecto Food Facility Honduras.

SDDE y FAO. (2018). Línea de Base para la reformulación del Plan Maestro de Abastecimiento de Alimentos de Bogotá de acuerdo con las dimensiones de Seguridad Alimentaria y Nutricional Secretaría Distrital de Desarrollo Económico. UTF / COL / 095 / COL Bogotá.

SDDE y Región Central. (2020). Lineamientos técnicos para avanzar hacia un sistema agroalimentario sostenible del Distrito Capital con perspectiva regional.

Secretaría Distrital de Planeación [SDP]. (2019, 17 de enero). Planes Maestros. http://www.sdp.gov.co/gestion-territorial/planes-maestros/generalidades

\section{ANEXOS}

\subsection{Listado de Abreviaturas}


CGIAR Consultative Group for International Agricultural Research

CONPES D.C. Consejo Distrital de Política Económica y Social del Distrito Capital

CORABASTOS Central de Abastos de Bogotá

DD Decreto Distrital

DTS-PMASAB Documento Técnico de Soporte del Plan Maestro de Abastecimiento de Alimentos y Seguridad Alimentaria de Bogotá

EAAB Empresa de Acueducto y Alcantarillado de Bogotá

EAE Evaluación Ambiental Estratégica

EEP Estructura ecológica principal

EIA Evaluación de Impacto Ambiental

FCD Factores Críticos de Decisión

MADS Ministerio de Ambiente y Desarrollo Sostenible

MAVDT Ministerio de Ambiente, Vivienda y Desarrollo Territorial (Actual MADS)

MMA Ministerio de Medio Ambiente de Chile

MML Metodología de Marco Lógico

ODS Objetivos de Desarrollo Sostenible

PACA Plan de Acción Cuatrienal Ambiental

PDD Plan de Desarrollo Distrital

PDSAN Política Distrital de Seguridad Alimentaria y Nutricional 2007-2015 (DD 508 de 2007)

PGA Plan de Gestión Ambiental del Distrito Capital

PMASAB Plan Maestro de Abastecimiento de Alimentos y Seguridad Alimentaria de Bogotá

POT Plan de Ordenamiento Territorial

PPP Política/Plan/Programa

SAS Sistema Ambiental Sectorial

SDDE Secretaría Distrital de Desarrollo Económico 